Abstract

\title{
BKTyper-Web Application for VP1 and NCCR Polyoma BK Typing ${ }^{+}$
}

\author{
Joan Martí-Carreras and Piet Maes* \\ Laboratory of Clinical and Epidemiological Virology, Department of Microbiology, Rega Institute, \\ Immunology and Transplantation, KU Leuven, Herestraat 49 Box 1040, BE3000 Leuven, Belgium; \\ joan.marti@kuleuven.be \\ * Correspondence: piet.maes@kuleuven.be \\ + Presented at Viruses 2020-Novel Concepts in Virology, Barcelona, Spain, 5-7 February 2020. \\ Published: 9 June 2020
}

\begin{abstract}
Human polyoma BK virus (BKV) prevalence has been increasing due to the introduction of more potent immunosuppressive agents, mostly in immunocompromised patients. BKV has been linked mostly to polyomavirus-associated hemorrhagic cystitis, and polyomavirus-associated nephropathy. BKV is a circular double stranded DNA virus (cdsDNA) with an average genome size of $5100 \mathrm{bp}$ and an average GC content of $40 \%$. Its genome codifies for five proteins: VP1, VP2, VP3, Angio gene, and the antigen $\mathrm{T}$ (which includes an event of alternative splicing, yielding a short and a large antigen $\mathrm{T}$ transcript). Additionally, it contains the non-coding control region (NCCR), known to be highly repetitive and to vary in number, length, and location of the repeats. Subtyping of BKV has been mainly studied in VP1 and the NCCR. Subtyping and subgrouping of BKV is conducted routinely in diagnostic assays and in epidemiological studies. Recently, Morel et al. published (Journal of Clinical Microbiology 2017; 55, 4) a strategy to subtype BKV through 100 bp VP1 amplicon. NCCR diversity is more complex than VP1, as it is configured by five repeat blocks $(\mathrm{O}, \mathrm{P}, \mathrm{Q}$, $\mathrm{R}$, and S). NCCR blocks can vary in number and length, resulting in a gradient of infectivity and replication. Rearranged NCCR have been linked to diverse patient etiologies, although any specific arrangement has failed to correlate with disease outcome or to have any predictive value. Due to the high abundance of BKV individuals and the clinical implications for human health that may represent BKV typing, a reliable, automatic, and free typing tool would be of great interest. Here, BKTyper is presented, a whole genome genotyper for polyoma BKV, based on a VP1 typing by Morel's algorithm and NCCR block identification. BKTyper can accept both whole BKV genome or regions of interest in fasta format to generate the typing profile and phylogenetic analysis.
\end{abstract}

Keywords: human BK polyoma virus, VP1, BKTGR, NCCR

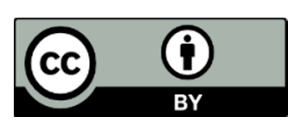

(C) 2020 by the authors. Licensee MDPI, Basel, Switzerland. This article is an open access article distributed under the terms and conditions of the Creative Commons Attribution (CC BY) license (http://creativecommons.org/licenses/by/4.0/). 